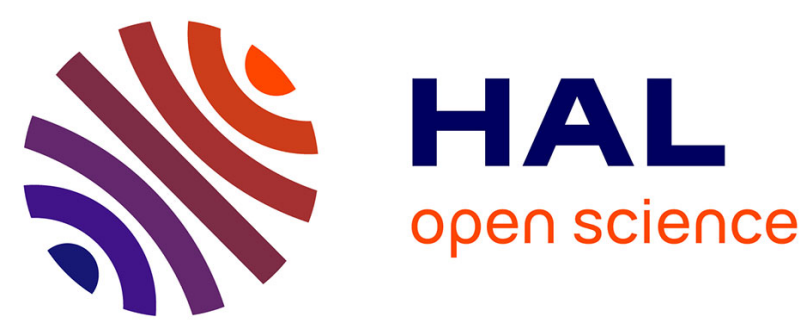

\title{
Monitoring gap junctional communication in astrocytes from acute adult mouse brain slices using the gap-FRAP technique
}

Chenju Yi, Jérémy Theillon, Annette Koulakoff, Hugues Berry, Christian Giaume

\section{To cite this version:}

Chenju Yi, Jérémy Theillon, Annette Koulakoff, Hugues Berry, Christian Giaume. Monitoring gap junctional communication in astrocytes from acute adult mouse brain slices using the gap-FRAP technique. Journal of Neuroscience Methods, 2018, 303, pp.103-113. 10.1016/j.jneumeth.2018.03.005 . hal-01734652

\section{HAL Id: hal-01734652 \\ https://hal.science/hal-01734652}

Submitted on 14 Mar 2018

HAL is a multi-disciplinary open access archive for the deposit and dissemination of scientific research documents, whether they are published or not. The documents may come from teaching and research institutions in France or abroad, or from public or private research centers.
L'archive ouverte pluridisciplinaire HAL, est destinée au dépôt et à la diffusion de documents scientifiques de niveau recherche, publiés ou non, émanant des établissements d'enseignement et de recherche français ou étrangers, des laboratoires publics ou privés. 


\title{
Monitoring gap junctional communication in astrocytes from acute adult mouse brain slices using the gap-FRAP technique
}

\author{
Chenju Yi ${ }^{1,2,3}$, Jérémy Theillon ${ }^{1,2,3}$, Annette Koulakoff ${ }^{1,2,3}$, Hugues Berry ${ }^{4,5}$ and \\ Christian Giaume ${ }^{1,2,3^{*}}$
}

\footnotetext{
${ }^{1}$ Collège de France, Center for Interdisciplinary Research in Biology (CIRB)/Centre National de la Recherche Scientifique, Unité Mixte de Recherche 7241/ Institut National de la Santé et de la Recherche Médicale U1050, 75231 Paris Cedex 05, France

${ }^{2}$ University Pierre et Marie Curie, ED, №158, 75005 Paris, France

${ }^{3}$ MEMOLIFE Laboratory of Excellence and Paris Science Lettre Research University, 75005 Paris, France

${ }^{4}$ INRIA, 69603 Villeurbanne, France

${ }^{5}$ University of Lyon, LIRIS UMR5205, 69622 Villeurbanne, France
}

Corresponding author:

Christian Giaume

CIRB Collège de France

11 place Marcelin Berthelot

75005 Paris, France

Tel: 33144271222

Fax: 33144271268

Email: christian.giaume@college-de-france.fr

Running title: gap-FRAP in adult acute hippocampal slices

Keywords: gap junctions, glia, connexins, fluorescence recovery after photobleaching 
Intercellular communication through gap junction channels plays a key role in cellular homeostasis and in synchronizing physiological functions, a feature that is modified in number of pathological situations. In the brain, astrocytes are the cell population that expresses the highest amount of gap junction proteins, named connexins. Several techniques have been used to assess the level of gap junctional communication in astrocytes, but so far they remain very difficult to apply in adult brain tissue. Here, using specific loading of astrocytes with sulforhodamine 101, we adapted the gap-FRAP (Fluorescence Recovery After Photobleaching) to acute hippocampal slices from 9 month-old adult mice. We show that gap junctional communication monitored in astrocytes with this technique was inhibited either by pharmacological treatment with a gap junctional blocker or in mice lacking the two main astroglial connexins, while a partial inhibition was measured when only one connexin was knocked-out. We validate this approach using a mathematical model of sulforhodamine 101 diffusion in an elementary astroglial network and a quantitative analysis of the exponential fits to the fluorescence recovery curves. Consequently, we consider that the adaptation of the gap-FRAP technique to acute brain slices from adult mice provides an easy going and valuable approach that allows overpassing this age-dependent obstacle and will facilitate the investigation of gap junctional communication in adult healthy or pathological brain. 


\section{Introduction}

Direct intercellular communication through gap junction channels plays a key role in cellular homeostasis and in synchronizing physiological functions (Laird et al., 2015). In the brain, this is the case for glial cells and in particular for astrocytes that exhibit the highest expression level of gap junction proteins, named connexins (Cxs) (Ransom and Giaume, 2013). Two major Cxs are expressed in astrocytes of adult mouse brain: $\mathrm{C} \times 43$, that is already detected at birth, and $\mathrm{C} \times 30$, that starts to be detected after the second postnatal week (Nagy and Rash, 2000). Such high expression level allows important intercellular exchanges of ions, signaling molecules and metabolites that provide the basis for a glial networking (Giaume et al., 2010). A specific feature of astrocytes is that they are located at the interface between neurons and blood vessels: indeed, they contact the synapses where they contribute to the "tripartite synapse" (Araque et al., 1999) and the vascular system since astrocyte endfeet are associated to the brain blood barrier (BBB). In addition, astrocytes are coupled with oligodendrocytes and can form panglial networks with them (Griemsmann et al., 2015). Interestingly, gap junction proteins are detected nearby cortical excitatory synapses (Genoud et al., 2015) and Cx43 as well as Cx30 are highly expressed at contacts between astroglial endfeets that enwrap blood vessels (Ezan et al., 2012). Accordingly, gap junctional communication (GJC) is involved in the control of synaptic transmission and in the maintenance of BBB integrity. Indeed, in Double KO mice for $\mathrm{C} \times 43$ and $\mathrm{C} \times 30$, synaptic transmission and plasticity are impacted in the hippocampus (Pannasch et al., 2011) and the integrity of the BBB is weakened (Ezan et al., 2012). Also, it is established that GJC in astrocytes is controlled by a number of endogenous compounds such as neurotransmitters, cytokines and endogenous lipids (Ransom and Giaume, 2013) and depends on neuronal activity (Roux et al., 2011; Liu et al., 2013). In addition, the expression level of astroglial $\mathrm{Cxs}$ is modified in a number of brain pathologies and injuries although the functional status of gap junction channels is much less documented (see Giaume et al., 2010). Hence, it appears essential to develop techniques to monitor the level of GJC in astrocytes at adult stages and in pathological situations to investigate the correlation between changes in $\mathrm{Cx}$ expression and function. 
So far, several methods have been used to monitor the level of GJC and its regulation in astrocytes studied in acute brain slices (see Giaume et al., 2012). This mainly includes approaches based either on electrophysiological properties of gap junction channels or on "dye coupling" experiments that consist in injecting into one astrocyte a membrane non-permeable molecule with a low molecular weight $(<700$ 800 Da such as Lucifer yellow, sulforhodamine B, biocytin, ...) that diffuses with time to neighboring coupled cells. In astrocytes, the double patch-clamp technique allows recording single-channel junctional currents in cultured cells (Giaume et al., 1991; Dermietzel et al., 1991) as well as monitoring macroscopic junctional currents in acute slices (Même et al., 2009; Zhong et al., 2016), while dye injections visualize astroglial and panglial networking (Blomstrand et al., 2004; Houades et al., 2006; 2008; Roux et al., 2011; Griemsmann et al., 2015). Alternatively, the intercellular diffusion of dyes can also by studied by adding Lucifer yellow in the external solution during the slicing procedure (Menezes et al., 2000) or by applying sulforhodamine 101 (SR101) either in vivo on the pial surface (Nimmerjahn et al., 2004) or by intravenous injection (Appaix et al., 2012). However, these methods suffer from some limitations. Although dual patch clamp recording and dye injections can be routinely performed in slices from juvenile animals, they become difficult in the adult. Alternatively, approaches based on dye loading, mainly SR101, after deposition on the pia or passage through the BBB can be used in vivo and in adults, are difficult to control from one experiment to the other. This strongly jeopardizes the comparison of GJC levels in different conditions. Interestingly, non-invasive techniques based on the gap-Fluorescent Recovery After Photobleaching (gap-FRAP) (Delèze et al., 2001) and its derivate the Local Activation of Molecular fluorescent probes (LAMP) (Yang and Li, 2009) have been developed in cultured cells. They consist in loading a population of coupled cells with a cell-permeable and photoactivatable fluorophore, then photolyzing locally the dye in one cell and recording fluorescence recovery. We have adapted the gap-FRAP technique to study astroglial GJC in acute hippocampal slices from old mice. Indeed experiments were performed at 9 months, an age at which very few analysis of GJC has been reported in the literature due to the difficulty to apply classical double patch-clamp recording or dye coupling experiments in acute brain slices (see Discussion). In order to load solely in astrocytes, we took advantage of sulforhodamine 101 (SR101), a fluorescent low molecular weight (607 Da) dye that is selectively taken up by astrocytes and passes through gap junction channels 
(Nimmerjahn et al., 2004; Schell et al., 2015).

In the present study, we demonstrate that the gap-FRAP technique can be adapted to acute hippocampal slices from old mice. GJC monitored in astrocytes with this technique was blocked either by pharmacological treatment or by genetic deletion of astroglial Cxs. Finally, we validate this approach using a mathematical model of SR101 diffusion in astrocyte networks. This model also suggested that such approach actually quantifies both intercellular and intracellular GJC. 


\section{Results}

A gene profile study from CNS cells has identified Aldh1L1 as an astroglial specific marker in the CNS (Cahoy et al., 2008). In the present study, adult Aldh1L1-eGFP transgenic mice were used to detect astrocytes in the CA1 area of the hippocampus (Fig. $1 A_{1}, B_{1}$ and $C_{1}$ ). Using 9 month-old mice, we found that eGFP-expressing cells in Aldh1L1-eGFP mice were always characterized by a soma size $<10 \mu \mathrm{m}$ and a typical rich arborization (Fig. $1 \mathrm{C}_{1}$ ) defining the so-called astroglial domain as previously reported (Yang et al., 2011; Zhong et al., 2016). In addition, no cell with a neuronal morphology was identified based on the presence of eGFP fluorescent signal. Similar observations were made using the fluorescent dye SR101 (Fig. $1 \mathrm{~A}_{2}$, $\mathrm{B}_{2}$ and $\mathrm{C}_{2}$ ) for astrocyte loading (Nimmerjahn et al., 2004). Consequently, as illustrated in figure 1, we examined the co-localization of eGFP with SR101 staining. Quantification of dual staining for eGFP and SR101 (Fig. $1 A_{3}, B_{3}$ and $C_{3}$ ) in the adult mouse hippocampus indicated that $93 \pm 1 \%$ ( $n=809$ cells from 4 mice) of SR101positive cells were eGFP-positive and $99 \pm 1 \%$ ( $n=754$ cells from 4 mice) of eGFPpositive cells were also SR101-positive. These numbers are in agreement with previous studies using SR101 staining in the hippocampus of young mice expressing eGFP driven either by hGFAP (Schnell et al., 2012) or Aldh1L1 (Zhong et al., 2016) (see also Nimmerjahn et al., 2004). Consequently, we concluded that SR101 is a reliable and specific marker for the identification and loading of adult astrocytes in the CA1 region, and that this marker can be used for gap-FRAP functional study of GJC in hippocampal astrocyte networks.

Initially, we carried out gap-FRAP experiments using the two-photon configuration of the confocal microscope. A SR101-positive astrocyte was targeted and a $5 \times 5 \mu \mathrm{m}$ square area was exposed to a laser illumination (5 flashes of $5 \mathrm{sec}$ each during 25 $\mathrm{sec})$. This procedure resulted in a bleaching of this astrocyte as indicated by a reduction in the recorded fluorescence level. With time, a recovery of fluorescence was observed and after $8 \mathrm{~min}$ it stabilized to a fluorescent level corresponding to $74 \pm$ $4 \%(n=6)$ of the basal fluorescence level recorded before bleaching (with a percentage of recovery $64 \pm 3 \%$ obtained by subtracting the background after bleaching) (Fig. $3 A_{1}$ and $A_{2}$ ). When the gap junction channel inhibitor carbenoxolone (CBX, $200 \mu \mathrm{M})$ was applied 15 min before, during and after the laser pulses, the level of fluorescence recovery was reduced by $24 \pm 3 \%, n=4$ (39 $\pm 3 \%$ of the recovery 
level compared to control condition) (Fig. $\left.3 \mathrm{~A}_{2}\right)$. These results indicated the occurrence of a reduction in GJC between the bleached astrocyte and its neighbors. However, as CBX used at this concentration is well known to totally abolish GJC in hippocampal astrocytes studied in acute slices (see for instance Wallraff et al., 2004; Rouach et al., 2008) the sensitivity of the two-photon configuration was not considered as satisfactory. In fact, in this configuration, the thickness of the laser illumination was very limited in the $Z$ axis (Fig. $2 A_{1}$ and $\left.B_{1}\right)$, hence the poor resolution of the GJC component in the recovery phase was interpreted as the consequence of a low proportion of SR101 bleached molecules in the targeted astrocyte compared to the total amount of SR101 molecules contained in its cytoplasm, including the soma and the primary processes (Fig. $2 \mathrm{~A}_{1}$ and $\mathrm{B}_{1}$ ).

In order to increase the proportion of SR101 molecules available for photolysis, we decided to use the same confocal microscope but in a one-photon configuration which results in a much larger thickness of the laser stimulation in the $Z$ axis (Fig. $2 A_{2}$ and $B_{2}$ ) and consequently in a higher number of SR101 bleached molecules, in particular in the soma. Moreover, a larger square area with the size $15 \times 15 \mu \mathrm{m}$ targeting a SR101-positive astrocyte was exposed to a laser illumination (15 flashes of $0.684 \mathrm{sec}$ each during $10.26 \mathrm{sec}$ ). By using the one-photon configuration, the recovery level in control condition is $65 \pm 3 \%(n=5)$, while it's only $23 \pm 4 \%(n=4)$ in presence of CBX, so the CBX-sensitive component is $42 \pm 3 \%(n=4)\left(\right.$ Fig. $3 B_{1}$ and $B_{2}$ ). Figure 4 illustrates examples of gap-FRAP experiments in SR101 loaded populations of hippocampal astrocytes using the one-photon configuration of the microscope. In control condition, after selection of a targeted SR101-positive astrocyte (Fig. 4A, left image) in the CA1 region, fluorescence disappeared after the laser bleaching in the soma (Fig.4A, middle image) and reappeared progressively with time after laser illumination (20 min, Fig.4A, right image). In contrast, when the slice was pre-incubated with CBX $(200 \mu \mathrm{M})$ the recovery of fluorescence monitored after bleaching was much weaker (sequential images in Fig. 4B taken at similar time than in Fig. 4A). Quantification of these two conditions is illustrated in figure 4C. We also noted that fluorescence recovery appeared faster with CBX than in control condition. Then we took advantage of knock out mice for the two major Cxs expressed in astrocytes, i.e. KO Cx43 and KO Cx30 (Ransom and Giaume, 2013), as well as the Double KO mice (KO for both $\mathrm{Cxs}$, see Methods) to obtain conditions 
in which GJC is partly or totally abolished. Using these animals we observed that, the recovery after photo-bleaching was reduced by $21 \pm 3 \%(n=12)$ for the KO Cx43, 15 $\pm 3 \%(n=10)$ for the KO Cx30 and $38 \pm 3 \%(n=9)$ for the Double KO compared to the recovery value in control condition, i.e. wild type. These values were determined by subtracting the plateau value measured at $\mathrm{t}=20 \mathrm{~min}$ after bleaching to the value measured immediately after bleaching. In this set of experiments the recovery for hippocampal slices from wild type mice was $65 \pm 3 \%(n=5)$ (Fig. 4D). It is noteworthy that the inhibition of fluorescence recovery was the same for CBX-treated wild type mice and the Double KO mice (Fig 4C and D). Altogether, these results indicate that gap-FRAP applied in the one-photon configuration allows assessing the level of GJC between astrocytes in various situations.

Moreover, after the initial bleaching of the fluorescent molecules in the targeted cell exposed to laser illumination, the neighboring reservoir cells are expected to give in return new fluorescent ones if the cells are coupled together by gap junction channels. This supposes that the level of fluorescence in the reservoir cells should decrease as fluorescence comes back in the bleached cell (see Delèze et al., 2001). This feature was qualitatively observed in gap-FRAP in SR101-loaded astrocytes from acute slices. Indeed, in astrocytes close to the bleached cell, fluorescence significantly decreased with time reaching $86 \pm 6 \%(n=9)$ of fluorescence, at $t=20$ min, compared to its level before illumination, while in astrocytes located far from the targeted cell, the change in fluorescence level was not detectable $99 \pm 1 \%(n=16)$ (Fig. 4E). In the classical gap-FRAP method initially described between two coupled cells, there is a direct relationship between the fluorescence recovery in the bleached cell and the donor cell (Déleze et al., 2001). In our case, the situation is more complex since we are dealing with a bleached cell and a network of donor cells; also the fluorescence recovery of the bleached cell is not completed after $1250 \mathrm{~s}$. Thus the rule of fluorescence loss in donor cells does not follow this direct relationship initially observed for a pair of cultured cells. This explains why the kinetics of fluorescence in donor cells continues to decrease while the fluorescence of the bleached cell reaches a plateau. In the presence of CBX, as expected, no change in the fluorescence was observed in astrocytes whether located close or far from the bleached cell ( $97 \pm 2 \%, n=6$ and $98 \pm 2 \%, n=5$, respectively) (Fig. 5F) while the 
rate of fluorescence recovery in this astrocyte was greatly reduced as described above (Fig. 5C).

In order to validate the-correctness of our interpretation of the gap-FRAP data and the analysis of GJC in astrocytes, we used a mathematical model based on morphological and experimental data. This mathematical model is based on SR101 diffusion in an elementary network. In agreement with the morphologies shown in figure 1, the photo-bleached cell (Cell 1 in figure 5A) was modeled as an object of $50 \mu \mathrm{m}$ total length, which included a $10 \mu \mathrm{m}$-large cell body flanked by two $20 \mu \mathrm{m}$ long processes on each side. These parameters were deduced from morphological analysis of stained astrocytes as illustrated in figures $1 \mathrm{C}_{1}$ and $\mathrm{C}_{2}$. Since we do not expect significant signal variation in the Z-axis during the experiments, we considered cells as two-dimensional objects. To emulate diffusion into the bleached astrocyte of SR101 molecules coming from nearby coupled astrocytes, we also position two "reservoir" cells (Cell 2 and Cell 2' in figure $5 \mathrm{~A}$ ) that are coupled to the extremities of Cell 1 processes via gap junctions. Note that the overall shape of Cells 2 and Cell 2' was kept as simple as possible to limit computation times. As a starting point, we used the experimental data obtained in the presence of CBX (Fig. 4C), i.e. without GJC, to estimate the SR101 diffusion coefficient $D_{\mathrm{cbx}}$ and the soma surface area of the bleached cell (Cell 1), $v_{\mathrm{S}}$. Figure 4F shows that in the presence of CBX the fluorescence level in astrocytes around the bleached cell is not altered by the photo-bleaching process, even for those that are found close to the bleached cell. Therefore we considered that in the presence of CBX, GJC between astrocytes is totally abolished, in agreement with the well-documented effect of CBX on GJC in acute slices (Wallraff et al., 2004; Rouach et al., 2008). We thus fixed the coupling strength $G$ in the mathematical model to $G=0$ and used nonlinear optimization to estimate the values of $D_{\mathrm{cbx}}$ and $v_{\mathrm{S}}$ based on the experimental data of Fig. $4 \mathrm{C}$. We estimated $D_{\mathrm{cbx}}=1.35 \mu \mathrm{m}^{2} / \mathrm{s}$ and $v_{S}=70 \mu \mathrm{m}^{2}$. As illustrated in figure $5 \mathrm{~B}_{1}$, the initial reduction of fluorescence in the $\mathrm{ROI}$ after photo-bleaching progressively recovered due to fluorescent SR101 molecules coming from the processes of the photobleached cell itself but not from the reservoir cells, as expected. Note that in this case the resulting quality of the fit between the curve obtained from the model and the experimental data was excellent (Figure $\left.5 \mathrm{~B}_{2}\right)$. When applied to the gap-FRAP data in the Double KO mice (Figure 4D), we obtained similar results $\left(D_{\text {doublekO }}=1.00 \mu \mathrm{m}^{2} / \mathrm{s}\right.$ 
and $v_{S}=50 \mu \mathrm{m}^{2}$ ), with, again an excellent fit to the data (Figure $5 \mathrm{~B}_{2}$ ). We then used the experimental gap-FRAP data obtained in control condition (Fig. 4C) to estimate the SR101 diffusion coefficient in control condition $D_{\text {ctrl }}$; the surface area of each reservoir cells $V_{R}$ and the coupling strength $G$. In this case, we fixed the soma surface area of the bleached cell to the value estimated with CBX condition indicated above, i.e. $v_{\mathrm{S}}=70 \mu \mathrm{m}^{2}$. Our estimations yielded: $D_{\text {ctrl }}=3.04 \mu \mathrm{m}^{2} / \mathrm{s}, G=1.0$ and $v_{\mathrm{R}}=105$ $\mu \mathrm{m}^{2}$, with excellent fit to the experimental data (Figure $5 \mathrm{C}_{2}$ ). Unlike the CBX case, the initial reduction of fluorescence in the ROI after photobleaching now recovered both by fluorescence coming from the processes of the photo-bleached cell and from the reservoir cells. This is evidenced in Figure $5 \mathrm{C}_{1}$ where the color intensity of both the processes of Cell 1 and that of Cell 2 and Cell 2 ' decreased during fluorescence recovery while intensity in the $\mathrm{ROI}$ increased. The estimated apparent diffusion coefficient $D$ is therefore reduced by a factor of 2.2 with $\mathrm{CBX},\left(D_{\mathrm{ctrl}}=3.04\right.$ vs $D_{\mathrm{cbx}}=1.35$ $\mu \mathrm{m}^{2} / \mathrm{s}$.). We note that such a difference between the apparent diffusion coefficients (ctrl vs CBX) was consistently obtained in our fits to the experimental data. For instance, we could not get good fitting quality when the two diffusion coefficients (ctrl and $(B X)$ were constrained to adopt identical values. Hence, our study indicates that CBX reduces the apparent diffusion coefficient of SR101 within the processes of the photo-bleached cell.

When applied to $\mathrm{C} \times 30 \mathrm{KO}$ and $\mathrm{Cx} 43 \mathrm{KO}$, the estimates from our mathematical model for the intercellular coupling strength $G$ were clearly nonzero but less than 1.0 ( $G=0.27$ and 0.50 , respectively). This suggests that intercellular GJC is severely impaired in Cx30 KO and Cx43 KO hippocampal astrocytes as previously reported (see Rouach et al., 2008). This is in strong contrast with the value estimated in control conditions above, $G=1.0$. The interpretation of $G=1.0$ in the model is that the rate of intercellular diffusion of SR101 is not significantly different from its intracellular diffusion rate. This suggests that in control condition, the targeted astrocyte and its coupled neighbors can be considered as a unique cell from the point of view of SR101 diffusion, this is directly due to the well-known very high level of GJC in hippocampal astrocytes. Indeed, astroglial networking can involve about 80 to more than 100 astrocytes when biocytin is used as an intercellular tracer (Blomstrand et al., 2004; Zhou et al., 2010; Strohschein et al., 2011). In contrast, in Cx30 KO and Cx43 KO, reduction of GJC strength would partially disrupt the astroglial networking. 
We tested this idea using non-linear fitting of the gap-FRAP curves. In the case of an isolated astrocyte, with no coupling to any other cell, one expects fluorescence recovery to exhibit 1-component exponential kinetics, since fluorescence recovers from a single, intracellular source of SR101. However, when the astrocyte is coupled to other cells, fluorescence recovers from two sources (intracellular SR101 and the SR101 initially present in the coupled cells), and one expects a 2-component kinetics with a fast component due to the intracellular SR101 diffusion and a slower one due to the intercellular SR101 diffusion. We thus fitted each gap-FRAP curves with 1component or 2-component exponential functions and used Akaike Information Criterion to compare the quality of the fits (see Methods). Figure 6A shows the fraction of cells in each condition for which the best model was found to be 1component (white) or the 2-component (black) kinetics. With CBX, we found that the best model is 1-component kinetics for most of the cells, in agreement with our hypothesis above. Marginal improvements of the 2-component fits are not enough to compensate for the larger number of fitting parameters (Fig. 6B). We interpret the corresponding time constant of the single exponential (referred to as the "time scale" below) $K_{\mathrm{cbx}}=0.00147 \mathrm{~s}^{-1}$ as the time scale for intracellular diffusion of SR101 (see Table 1). In control conditions however, the 2-component scenario is the best fit for all our experiments (Fig. 6C), again as expected. A minor fraction of recovery comes from a fast component with estimated time scale $K_{\text {ctrl1 }}=0.00362 \mathrm{~s}^{-1}$ (Table 1), whereas a larger recovery fraction is due to a component that is almost tenfold slower $\left(K_{\text {ctrr }}=0.00049 \mathrm{~s}^{-1}\right)$. Considering the fraction of recovery associated with each component, we associate the fast component with intracellular diffusion and the slower one with intercellular diffusion. Comparing the values of the time scales between CTRL and CBX is difficult because they result from different fitting models. However, when we compare $K_{\mathrm{ctrl} 1}$ and $K_{\mathrm{cbx}}$, the decrease due to CBX is similar to the reduction of the apparent diffusion coefficient suggested by our mathematical model above $\left(K_{\mathrm{ctrl} 1} / K_{\mathrm{cbx}}=2.5\right.$ whereas $\left.D_{\mathrm{ctrl}} / D_{\mathrm{cbx}}=2.2\right)$. We therefore conclude that this analysis confirms the decrease of apparent diffusion kinetics with CBX.

For the two KO models (CX30 and CX43), fluorescence recovery is almost always best fitted with a 2-component exponential (Fig. 6A and D). Statistical analysis of the parameters (Table 1) shows that in both cases the two time scales and the fraction of 
recovery associated with the fast intracellular component are not different from control conditions. The only statistically significant difference is the fraction of recovered fluorescence associated with the slow intercellular component (Amplitude $)_{2}$ ) that is between 60 and $70 \%$ of the control value. We note that this decay is of the same magnitude as the decrease of the intercellular coupling strength parameter $G$ in the $\mathrm{KO}$ astrocytes, obtained with our mathematical model above (between $27 \%$ and $50 \%$ ). Therefore, our analysis suggests that CX30KO and CX43KO astrocytes are coupled to a lower number (or a lower volume) of astrocytes, as intuitively expected.

The results with the Double KO CX30/CX43 are more complex. One half of our measured cells are best fitted with 1-component kinetics and the rest with 2component kinetics (Fig. 6A). The cells for which 1-component kinetics is the best exhibit the same kinetic parameters (recovery time scale and amplitude) as the CBX cells (Table 1). However, the Double KO cells that are best fitted with 2-component kinetics display kinetic parameters that shares common features with single KOs: compared to control, their fast intracellular kinetics are unchanged while the fraction of recovered fluorescence contributed by the slow intercellular component is much smaller. However, in addition, the time scale of the slow intercellular component of the Double KO is significantly larger, which is not the case for single KOs. Therefore, our analysis of the Double KO mutants indicates SR101 recovery kinetics that are intermediate between CBX and the single KOs. 


\section{Discussion}

We here report how the gap-FRAP technique, so far mainly used in cultured cell systems, can be adapted to assess gap junction-mediated communication in astrocytes studied in acute hippocampal slices from the adult mouse. Its accuracy to detect changes in GJC was demonstrated by using pharmacological as well as genetic tools to reduce or abolish GJC in astrocytes. In addition, we carried out a data fitting and model comparison that validated experimental data and interpretation. The development of this approach provides an easy going technique that allows assessing the level of GJC in astrocytes at late ages, a feature that was so far difficult to achieve and was time consuming when using classical dye coupling experiments in adult acute brain slices. However, as it has been reported (Schnell et al., 2012) the active uptake of SR101 by astrocytes depends on brain areas; such feature should thus be taken into account for GJC measurement by gap-FRAP technique in regions other than the hippocampus.

Up-to-now the sole work reported to use gap-FRAP technique in acute slices from aged brain was performed using CDCF (dicarboxy-dichlorofluorescein diacetate), a gap junction channel- and cell membrane-permeable dye that becomes membraneimpermeable after de-esterification (Cotrina et al., 2001). This study reported that while the expression of $\mathrm{C} \times 43$ and $\mathrm{C} \times 30$ is rather stable, according to age, GJC tends to decrease. However CDCF, based on its permeability properties, is expected to enter all cells, which is certainly a handicap in a tissue like the brain composed of a variety of cellular populations including neurons, glial and endothelial cells. Thus, although in adult mouse the main cell population connected by gap junctions is the astrocytes, it cannot be assumed that CDCF recovery after bleaching is only mediated by GJC between these cells. We overpassed this problem by using SR101, a dye that is specifically up-taken by astrocytes thanks to an active transport (Schnell et al., 2012) and passes through gap junction channels (Nimmerjahn et al., 2004). We verified that this was the case in 9 month-old hippocampal slices and thus considered to have in hand a method in which solely astrocytes were loaded. We have also been careful to avoid the secondary staining of oligodendrocytes that starts to be detected after 40 min (Hagos and Hulsmann, 2016; Hill and Grutzendler, 2014). Indeed, this astrocyte-to-oligodendrocyte coupling occurs through heterotypic gap junctions forming a panglial network that is, however, minor in the hippocampus 
compared to other brain region like the thalamus (Griemsmann et al., 2014). In our experimental condition, the use of the Aldh1L1-eGFP mouse indicated that more than $93 \%$ of the SR101 loaded cells expressed eGFP and thus were identified as astrocytes. Moreover, it has also been reported that low concentration $(1 \mu \mathrm{M})$ of SR101 induces a direct effect on pyramidal neuron membrane structures, leading to a reduction in action potential firing threshold, and a long-term increase in neuronal excitability and synaptic efficacy (Kang et al., 2010; Garaschuk et al., 2013). So, we do not exclude that in our experiments such SR101-induced effects on neuronal activity occurred. However, we have previously shown that in the CA1 region of the hippocampus, dye coupling (tested with sulforhodamine B and biocytin) is not controlled by changes in neuronal activity including epileptic-like discharges or TTX application (Rouach et al., 2008). Consequently, we consider that the gap-FRAP technique provides a trustable assessment of GJC in adult hippocampal slices and can be used to determine whether GJC is affected in different pathological situations as recently applied in a murine model of Alzheimer's disease (Yi et al., 2016).

By using pharmacological treatment with $\mathrm{CBX}$ or transgenic animals lacking the two major astroglial Cxs, the Double KO mouse, we demonstrated that the gap-FRAP technique was able to detect the total inhibition of GJC with a reduction of about $39 \%$ and $38 \%$ compared to control, respectively. The residual resting fluorescence was interpreted as the return of non-bleached molecules of SR101 from distant processes of the targeted astrocyte located outside the bleached zone. Based on this statement, it is expected that, using CDCF for cell loading (see Cotrina et al., 2001), this component that will also include neuronal processes and endothelial cells present in the bleached zone, will be largely increased and thus will reduce the signal-to-noise ratio. Using single astroglial $\mathrm{Cx}$ knock out, $\mathrm{Cx} 43 \mathrm{KO}$ and $\mathrm{C} \times 30 \mathrm{KO}$, we demonstrated that the gap-FRAP was enough sensitive to detect changes in the level of GJC. The lack of Cx43 was found to reduce by $21 \%$ fluorescence recovery compared to control condition, while the absence of Cx30 was associated to a $15 \%$ reduction. These data indicate that $\mathrm{C} \times 43$ contributed to $55 \%$ of GJC and $\mathrm{C} \times 30$ to $40 \%$. Interestingly, these changes in GJC associated to each $C x$ are very close to those reported previously using patch-clamp recording and dye coupling experiments in 3 week-old hippocampal slices which established that $\mathrm{Cx} 43$ and $\mathrm{CX} 30$ contribute to $50 \%$ and $35 \%$ of GJC, respectively, with the same single Cx knockout mice 
(Rouach et al., 2008). In addition, in another study (Wallraff et al., 2006) in the Cx43KO it was reported that Cx30 accounts for $50 \%$ of coupling. Finally, we demonstrated that while fluorescence recovered in the bleached astrocyte, those located close to this target cell loosed fluorescence while those located far did not show fluorescence loss. These features are expected when considering the processes of gap junction-mediated diffusion involved using the gap-FRAP technic (see Delèze et al., 2001).

A mathematical model based on experimental data obtained along our study was also carried out to validate the gap-FRAP technique. To reach this aim, we considered a two-dimension model that takes into account two compartments in the targeted cell, the central part that will be bleached and the distal processes that are out of this zone. Our model-based estimation of the apparent intracellular diffusion coefficient of SR101 in astrocytes is $3.0 \mu \mathrm{m}^{2} / \mathrm{s}$ in control conditions and 1.3 in CBXtreated cells. Albeit the diffusion coefficient of SR101 has thoroughly been measured in water (in the $[200,400] \mu \mathrm{m}^{2} / \mathrm{s}$ range, Gendron et al, 2008), quantitative studies of its value in living cells are very rare. In rod photoreceptors, SR101 diffusion coefficient was estimated to around $6 \mu \mathrm{m}^{2} / \mathrm{s}$ (Chen et al, 2002). Given that astrocyte processes are expected to be highly tortuous (Rusakov, 2015), our estimations fall within the expected range of values.

Inspecting the fitting of experimental recovery curves with those given by the model, we were able to distinguish two components in the recovery, one rapid and one slower. This was the case when using data from control (wild type), KO Cx43 and KO Cx30. In these cases, the rapid component was associated to the recovery due to SR101 molecules coming from the non-bleached distal processes of the targeted cells while the second component was associated to SR101 molecules contributed by adjacent cells through gap junction channels. The difference between $\mathrm{KO}$ and control mice was that in $\mathrm{KO}$ astrocytes, the slow intercellular component brought less SR101 in the bleached zone, thus confirming a weaker coupling. With the Double $\mathrm{KO}$, half of the cells exhibited exponential fit results identical to those obtained with $\mathrm{CBX}$, as intuitively expected. For the other half however, the fit results were close but not identical to single KOs. We cannot exclude that such discrepancy was due to alterations of morphology and physiology of Double KO astrocytes, reported as 
hypertrophic and reactive compared to wild type (Pannasch et al., 2011), a situation that was not taken into account in our model. However, it should be kept in mind that in the Double KO the lack of coupling is permanent during all animal life, while CBXinduced uncoupling only results from short time exposure and in this case morphological changes have not been studied so far. Finally, the analysis of recovery and our mathematical model indicated that the apparent diffusion coefficient of SR101 within the distal processes of the photo-bleached cell is strongly reduced in the presence of CBX. Our interpretation is that this reduction of apparent diffusion is caused by a global decrease of the intracellular tortuosity of the astrocytes with respect to SR101. We can however only speculate on the cause of such a CBXinduced increase of intracellular tortuosity. For instance, this could be due to CBXinduced changes in the cell or process morphology. Alternatively, it could be due to the inhibition by $\mathrm{CBX}$ of reflexive gap junctions, i.e. the gap junctions between processes belonging to the same astrocyte. Indeed, the occurrence of gap junctions between astroglial processes of the same cell has been evidenced by electron microscopy data (Wolff et al., 1998; Genoud et al., 2015);

There is increasing evidence that $\mathrm{Cxs}$ in astrocytes may play a role in several models of brain pathologies. Interestingly their roles are at the moment more considered through their hemichannel function (Bennett et al., 2012; Bosch and Kielian, 2014; Davidson et al., 2013; Orellana et al., 2016; Kim et al., 2016) than through gap junctional channel function. We think that this lack is in part due to the difficulty to have a simple experimental approach to determine the level of astroglial GJC in adult brain. Consequently, we consider that the adaptation of the gap-FRAP technique to acute brain slices from adult mice should now allow overpassing this technical obstacle. Indeed, the combination of approaches to assess hemichannel and gap junction channel functions of astroglial Cxs will contribute to fully investigate and understand their role in brain pathologies. 


\section{Experimental procedure}

All experiments were performed according to the European Community Council Directives of January first 2013 (2010/63/EU) and followed the Institute National de la Santé et de la Recherche Médicale (INSERM) guidelines for ethical treatment of animals. Experiments were also done in accordance with the institutional French (Comité Opérationnel pour l'Ethique dans les Sciences de la Vie du Centre National de la Recherche Scientifique, CNRS) and the international (NIH guidelines) standards and legal regulations (Ministère de l'Agriculture et de la Pêche) for the use and care of animals. Along this work all efforts were made to minimize the number of animals used and their suffering.

\section{Reagents}

All drugs were from SIGMA while Sulforhodamine 101 was obtained from ThermoFisher Scientific.

\section{Acute brain slices}

Acute brain hemisphere slices $(300 \mu \mathrm{m})$ from 9 month-old C57BI6, Cx30 ${ }^{(--)}$), (KO Cx30) (Dere et al., 2003), Cx43 (fl/f): hGFAP-Cre (KO Cx43) (Theis et al., 2003), Cx30 ${ }^{(-1-}$ ${ }^{\prime} \mathrm{C} \times 43^{(\mathrm{f} / \mathrm{fl})}$ :GFAP-cre (Double KO) (Wallraff et al., 2006) and Aldh1L1-eGFP (Yang et al., 2011) mice were cut on a vibratome (Microm HM 650V) and transferred to a holding chamber where they rested on a nylon mesh, submerged in oxygenated artificial cerebro-spinal fluid (ACSF) at room temperature for a stabilization period of 60 min. The ACSF solution contained (in $\mathrm{mM}$ ): $\mathrm{NaCl}$ (125); $\mathrm{KCl}$ (2.5); $\mathrm{NaHCO}_{3}$ (25); $\mathrm{NaH}_{2} \mathrm{PO}_{4}$ (1.25); glucose (25); $\mathrm{MgCl}_{2}$ (1); $\mathrm{CaCl}_{2}$ (2).

\section{Gap-Fluorescence Recovery After Photobleaching (gap-FRAP)}

Acute brain hemisphere slices of 9 month-old mice were prepared as described above and then were incubated with ACSF containing SR101 $(1 \mu \mathrm{M})$ at $37^{\circ} \mathrm{C}$ for 30 min. After 20 min wash in ACSF, slices were placed in a perfusion chamber on the stage of a confocal microscope and perfused with oxygenated ACSF during the entire experiment. Acquisitions were performed using a $25 \times(\mathrm{NA}=0.95)$ Leica water immersion objective hold on a Leica SP5 microscope controlled by the Leica acquisition software. The gap-FRAP experiments consisted in a three parts illumination sequence. During the initial part, the baseline fluorescence was collected 
every $25 \mathrm{sec}$ during $3 \mathrm{~min}$ on a $196.9 \times 196.9 \mu \mathrm{m}$ field. Laser power was set so that no bleaching of the SR101 happened. This power was $1 \mathrm{~mW}$ in the single-photon experiments and $67 \mathrm{~mW}$ in the two-photon experiments. Next, in order to photobleach the cell body of the target astrocyte, the laser scanning area was restricted to a smaller field ( $5 \times 5 \mu \mathrm{m}$ and then $15 \times 15 \mu \mathrm{m}$, see Results section) and 15 pulses of light were applied. The power applied was $3 \mathrm{~mW}$ in the single-photon experiment and $67 \mathrm{~mW}$ in the two-photon experiment. Then, the fluorescence recovery was recorded during 3 different time courses, one image immediately after bleaching, 10 images every $5 \mathrm{sec}$ and then every $25 \mathrm{sec}$ during $20 \mathrm{~min}$. Same field size and laser power were used as in the baseline acquisition. All laser power measurements were done by placing a power sensor at the objective turret, without an objective in place. We used the bleach-point function of the Leica acquisition software in order to avoid scanning and laser blanking during power measurement. Images were analyzed off-line: the mean fluorescence intensity $\left(F_{0}\right)$ of the astrocyte cell body was measured with Image $J$ software. $F_{0}$ was obtained from the first value of the baseline recording. The fluorescence intensity of each time point was normalized to $F_{0}$ and then expressed as percentage of $F_{0}\left(F_{t} / F_{0} \%\right)$. The percentage of recovery was calculated by using the plateau value (the average of the last 6 values of the recording) subtracting the value of the first time point after photobleaching. All studies were performed in the CA1 region of the hippocampus at a depth of $45 \mu \mathrm{m}$ below the surface to ensure that the cells of interest had not been damaged during the slicing procedure.

\section{Microscope settings}

Confocal images were acquired on a Leica SP5 confocal scanning unit installed on a DM6000CFS upright confocal stand. We used the HCX IRAPO, L 25x, NA=0.95, water immersion objective for all images. The acquisition software was the Leica Application Suite Advanced Fluorescence (LASAF) that provided all the needed options for realizing GAP-FRAP experiments. During all acquisitions (pre- and postbleach as well as bleach itself) the scanning speed was set to $400 \mathrm{~Hz}$ and image format to $512 \times 512$ pixels which is equivalent to a pixel dwell time of $4.88 \mu \mathrm{s}$. Field size was set to $196.9 \times 196.9 \mu \mathrm{m}$ for pre- and post- bleach. Field size was reduced to $5 \times 5 \mu \mathrm{m}$ or $15 \times 15 \mu \mathrm{m}$ in order to improve bleaching efficiency. 


\section{Mathematical model}

We modeled intracellular diffusion of SR101 in a two-dimensional model (Fig. 5A) featuring the bleached astrocyte (Cell 1 in the figure) and its gap junction-mediated coupled nearby astrocytes (Cells 2 and Cell 2'). We discretized the intracellular space of the cells with a square Euclidean lattice with spacing $\Delta x=\Delta y=1 \mu \mathrm{m}$. The lattice was used to solve numerically the diffusion equation: $\partial_{t} u(x, y, t)=D \nabla^{2} u(x, y, t)$ where $u(x, y, t)$ represents SR101 concentration at location $(x, \mathrm{y})$ and time $t$ and $D$ is the apparent diffusion coefficient of SR101 in the cell. Numerical resolution was done with a semi-implicit (Crank-Nicolson) finite volume scheme with Von Neumann boundary conditions and time step $\Delta t=0.1 \mathrm{~s}$.

In the model, SR101 diffusion between the bleached cell (Cell 1) and its two neighbors (Cell 2 and 2') occurred exclusively at the contact point between the extremity of Cell 1 processes and its neighbors. To model gap-junction coupling (GJC), the SR101 flux between the cells at these contact points was set to $J=-$ $G D \nabla u(x, y, t)$ where $G \in[0,1]$ is the strength of $\mathrm{GJC}$ relative to the intracellular diffusion of SR101. Therefore, we modeled GJC as a diffusive type of coupling but with possibly slower kinetics than intracellular cytosolic diffusion (i.e. $G<1$ ). Note that $G=$ 0 corresponds to non-coupled cells.

In agreement with our experimental setup (Fig. 2A2 -B2), the region of interest (ROI) i.e. the zone where fluorescence is monitored and/or bleached, was defined as the $16 \mu \mathrm{m}$-long area centered on the cell body and comprising in addition the first $3 \mu \mathrm{m}$ of the processes on each side. During numerical simulation, we compute the total SR101 amount present in the ROI at time $t$ and normalize it with the corresponding quantity before photobleaching, thus yielding $F I^{\text {model }}\left(t_{i}\right)$. We estimated from the experimental data the following parameters: the strength of GJC between the bleached cell and the coupled astrocytes $G$; the SR101 apparent diffusion coefficient $D$; the surface area of the soma of the bleached cell $v_{S}$ and the surface area of each reservoir astrocyte $v_{R}$. We used nonlinear optimization through evolution strategy (CMA-ES, Hansen, 2006) to find the parameter values that minimize the fitting error $\varepsilon$ $=\Sigma_{i=1 . . N}\left[F I^{\text {model }}\left(t_{i}\right)-F I^{\exp }\left(t_{i}\right)\right]^{2}$ where $F I^{\exp }\left(t_{i}\right)$ is the experimental value of the normalized fluorescence intensity in the ROI at the $i^{\text {th }}$ time point and $N$ the number of time points 
in the time series. For consistency, we constrained the estimated value of $G$ to remain in $[0,1]$ during optimization. We also checked that optimization systematically converged to the same optimal values whatever the initial guesses.

\section{Data fitting and model comparison}

Each GAP-FRAP curve was successively fitted with a 1-component exponential model: $Y(t)=Y_{\max }-\left(Y_{\max }-Y_{\min }\right) \exp (-K t)$ and with 2-component exponential model: $Y(t)=Y_{\max 1}-\left(Y_{\max 1}-Y_{\min } / 2\right) \exp \left(-K_{1} t\right)+Y_{\max 2}-\left(Y_{\max 2}-Y_{\min } / 2\right) \exp \left(-K_{2} t\right) . Y_{\min }$ was not fitted but fixed to the value of the fluorescence intensity immediately after bleaching (where $t$ $=0$ by convention). These fitting functions define the recovery time scales of each component $\left(K\right.$ or $K_{1}$ and $K_{2}$ ) and the fraction of recovery contributed by each component, i.e. its amplitude $\left(Y_{\max }-Y_{\min }\right.$ or $Y_{\max 1}-Y_{\min } / 2$ and $\left.Y_{\max 2}-Y_{\min } / 2\right)$. Since the number of parameters to be estimated doubles in the 2-component model, we compared the qualities of the resulting fit using the Akaike Information Criterion with a correction for finite sample sizes (Burnham and Anderson, 2004): $A I C_{c}=N \ln \left(\sigma^{2}\right)+$ $2 k+2 k(k+1) /(N-k-1)$, where $N$ is the number of data points of the time series, $k$ is $1+$ the number of parameters to be estimated and $\sigma^{2}=1 / N \Sigma_{i=1 . . N}\left[Y\left(t_{i}\right)-F I^{\exp }\left(t_{i}\right)\right]^{2}$, the mean estimated residuals from the fitted model. The best model is the one with the minimum $A I C_{c}$ value.

\section{Statistical analysis}

All data are presented as the mean \pm SEM. Comparisons between groups were conducted using unpaired t-test, except the comparison between groups "close" and "far" by two-way ANOVA, and $\mathrm{P}<0.05$ was considered to indicate a statistically significant difference. GraphPad Prism 6.0 software (GraphPad Software Inc., La Jolla, CA, USA) was used for graph processing. 


\section{Figure Legends}

Figure 1: Co-localization of $\mathrm{eGFP}^{+}$cells with the astroglial marker SR101 in the hippocampus CA1 of adult Aldh1L1-eGFP mice.

Representative images showing Aldh1L1-eGFP ${ }^{+}$cells (green), SR101 labelled astrocytes (red) and merged (yellow) within the CA1 region, A1-A3, at low magnification scale bar, $30 \mu \mathrm{m}$; B1-B3, at higher magnification, scale bar, $10 \mu \mathrm{m}$; C1C3, at high magnification showing the morphology of a single eGFP-expressing cell (green), SR101 labelled astrocyte (red) and their merge (yellow), scale bar, $2 \mu \mathrm{m}$.

Figure 2: Larger bleaching area in the targeted astrocyte by using one-photon microscopy in FRAP experiments

A1, B1, using the two-photon configuration of the confocal microscope, a $5 \times 5 \mu \mathrm{m}$ square area (shown in B1 left using a reference slide, scale bar $8 \mu \mathrm{m}$ ) in one SR101positive astrocyte was targeted and exposed to laser illumination ( 5 flashes of $5 \mathrm{sec}$ each for $25 \mathrm{sec}$ ); the thickness of the laser illumination illustrated in A1 was limited in the $Z$ axis ( $B 1$ right scale bar $8 \mu \mathrm{m}$ ); $A 2$, B2, using a one-photon configuration, $a$ larger square area of $15 \times 15 \mu \mathrm{m}$ (B2 left scale bar $20 \mu \mathrm{m}$ ) targeting a SR101positive astrocyte was exposed to laser illumination (15 flashes of $0.684 \mathrm{sec}$ each for $10.26 \mathrm{sec}$ ); this results in a much larger thickness of laser stimulation in the $Z$ axis (A2 and B2 right, scale Bar $20 \mu \mathrm{m}$ ) and consequently, also in a higher number of SR101 bleached molecules especially in the soma.

Figure 3: gap-FRAP experiments using the two-photon vs one-photon microscope A1, Curves showing time-lapse percentage of fluorescence intensity ( $F I)$ normalized to $F_{0}$ in the astrocytes of 9-month WT mice in control conditions (CTL, in black) and in $\mathrm{CBX}$ conditions ( $\mathrm{CBX}$, in red) by using a 2-photon microscope; A2, Bar histogram illustrates the quantification of fluorescence recovery percentage measured at $8 \mathrm{~min}$ after photobleaching; ( ${ }^{*} \mathrm{p}<0.05$, unpaired Student's $t$-test, mean \pm SEM, CTL $\mathrm{n}=6$, CBX $n=4) ; B 1$, Curves showing a single FRAP experiment by using a one-photon microscope B2, Quantification of the fluorescence recovery percentage measured at 20 min after photobleaching $\left({ }^{* * *} p<0.001\right.$, unpaired Student's $t$-test; mean \pm SEM, CTL $n=5 ; C B X n=4$ ) 
Figure 4: gap-FRAP experiments in SR101 loaded hippocampal astrocytes Fluorescence images showing astrocytes of acute brain slices from 9 month old WT mice loaded with SR101 before bleaching (left), immediately after bleaching in the white rectangle (middle), and $20 \mathrm{~min}$ after laser bleaching (right) in (A) control conditions (CTL) and in (B), under CBX conditions, scale bar, $20 \mu \mathrm{m}$; C, Curves showing the time-lapse percentage of fluorescence intensity $(\mathrm{FI})$ normalized to $\mathrm{F}_{0}$ in astrocytes of WT mice treated or not with CBX (mean \pm SEM, CTL $n=5 ; C B X n=4$ ); $D$, time-lapse percentage of normalized $\mathrm{FI}$ in the astrocytes from 9-month WT, Cx43KO, Cx30KO and Double KO mice (mean \pm SEM, WT $n=8$; Cx43KO n=12, Cx30KO $n=10$ and DKO n=9); E, time-lapse percentage of normalized $\mathrm{FI}$ in bleached astrocytes from WT mice (black), and in the astrocytes close (green) or far from the bleached cell (blue) ${ }^{* * *} \mathrm{p}<0.001$, Close vs Far, Two-way ANOVA, mean \pm SEM, CTL $\mathrm{n}=5$; Close $\mathrm{n}=9$; Far $\mathrm{n}=16$ ); $\mathrm{F}$, time-lapse percentage of normalized $\mathrm{FI}$ in bleached astrocytes from WT mice treated with CBX (red), and in the astrocytes close (green) or far from the bleached cell (blue) (mean \pm SEM, CBX $n=4$; Close $n=6$; Far $n=5$ ).

Figure 5: A mathematical model validates our interpretation of GAP-FRAP experiments.

(A) Layout of the two dimensional model used for numerical simulations of GAPFRAP: the bleached cell (Cell 1 , blue) is made of a central cell body $\left(10 \times 7 \mu \mathrm{m}^{2}\right)$ surrounded by two thinner process compartments $\left(20 \times 1 \mu \mathrm{m}^{2}\right.$ each). Cell 1 is coupled to two flanking cells (Cell 2 and 2', green) via gap junctions (GJ) located at the contact point between the extremities of Cell 1 processes and the flanking cells. The intracellular space is discretized by a square lattice, on which we solve numerically the diffusion equation to simulate SR101 intracellular and intercellular diffusion (see Experimental Procedure). The output of this mathematical model is shown $(B)$ in the presence of $\mathrm{CBX}$ or with the Double $\mathrm{KO}$ mice or $(C)$ in control conditions. Figures $\mathrm{B} 1$ \& C1 show the spatial distribution of SR101 in the cells at the indicated time point, using the indicated colormap. At time $t=0$, a central section of Cell 1 (the ROI), encompassing the cell body plus part of the processes (dashed rectangles) is photobleached. The simulation then monitors the recovery with time of the total fluorescence in the ROI, normalized by its value before photobleaching (B2-C2). After adjustment of the values of the model parameters $G$ (strength of the GJ- 
coupling between Cell 1 and 2 or 2) and $D$ (diffusion coefficient of SR101), the match between the model and the experimental data is excellent in the three conditions (B2C2). Parameter estimates were: i) $C B X: v_{S}=70 \mu \mathrm{m}^{2}, D=1.35 \mu \mathrm{m}^{2} / \mathrm{s}, v_{\mathrm{R}}=105 \mu \mathrm{m}_{2}$, i) Dble KO: $v_{\mathrm{S}}=50 \mu \mathrm{m}^{2}, D=1.00 \mu \mathrm{m}^{2} / \mathrm{s}$ and iii) Ctrl: $v_{\mathrm{S}}=70 \mu \mathrm{m}^{2}, D=3.04 \mu \mathrm{m}^{2} / \mathrm{s}, v_{\mathrm{R}}=105$ $\mu \mathrm{m}^{2}, G=1.0$.

Figure 6: Comparison of 1-component and 2-component fits for fluorescence recovery.

Each GAP-FRAP curve was fitted with 1-component exponential and 2-component exponential functions. The Akaike Information Criterion was used to decide whether one of the two functions yielded a better fit of the data-The fraction of cells for each case is shown as histograms in $(A)$. The number above each bar gives the total number of cells tested for each experimental condition or cell type. Illustration of the corresponding fits are given in $(B-D)$ with empty circles for experimental data (gapFRAP curve for a single cell), full black lines for fits with 2-component kinetics, and dashed red lines with 1-component kinetics.

Table 1: Statistical analysis of the exponential fits. For each condition, the numbers shown are the estimated mean \pm SEM of the two or four parameters of the 1component or 2-component exponential fits, respectively. For the double $\mathrm{KO}$ mice (CX30/CX43) we segregated the cells into two groups (1-component, 2-component), depending on what was their best fit, and analyzed each group separately. $n$ indicates the total number of cells for each case. The italicized numbers below the estimated parameter values give the $p$-value for hypothesis testing of the mean (homoscedastic $t$-test, two-tailed). All 2-component fits (CX30KO, CX43KO and DBLEKO 2-components) were tested against CTRL, whereas DBLEKO 1-component was tested against $\mathrm{CBX}$. 
Acknowledgements: The authors thank the animal facility staff of the Center of Interdisciplinary Research in Biology of the Collège de France and Pascal Ezan for technical help. Financial support was provided to CG by the Agence Nationale pour la Recherche grant NarConX 14-CE16-0022 and the Ligue Européenne Contre la Maladied'Alzheimer. 


\section{References}

- Appaix F, Girod S, Boisseau S, Römer J, Vial JC, Albrieux M, Maurin M, Depaulis A, Guillemain I, van der Sanden B. Specific in vivo staining of astrocytes in the whole brain after intravenous injection of sulforhodamine dyes. PLoS One. 7:e35169.

- Araque A, Parpura V, Sanzgiri RP, Haydon PG. (1999) Tripartite synapses: glia, the unacknowledged partner. Trends Neurosci. 22:208-215.

- Blomstrand F, Venance L, Sirén AL, Ezan P, Hanse E, Glowinski J, Ehrenreich H, Giaume C. (2004) Endothelins regulate astrocyte gap junctions in rat hippocampal slices. Eur J Neurosci. 19:1005-15.

- Burnham KP, Anderson DR (2004) Multimodel inference: understanding AIC and BIC in Model Selection. Socio. Meth. Res. 33: 261-304.

- Cahoy JD, Emery B, Kaushal A, Foo LC, Zamanian JL, Christopherson KS, Xing Y, Lubischer JL, Krieg PA, Krupenko SA, Thompson WJ, Barres BA. (2008) A transcriptome database for astrocytes, neurons, and oligodendrocytes: a new resource for understanding brain development and function. J Neurosci. 28:264-78.

- Chen C, Jiang Y, Koutalos Y (2002) Dynamic behavior of rod photoreceptor disks. Biophys J. 83(3): 1403-1412.

- Delèze J, Delage B, Henti-Ksibi O, Verrachia F, Hervé JC (2001) Fluorescence recovery after photobleaching. In "Connexin Methods and Protocols" Eds Bruzzone R and Giaume C. Humana Press Totowa, pp:313-27.

- Dere E, De Souza-Silva MA, Frisch C, Teubner B, Söhl G, Willecke K, Huston JP. (2003) Connexin30-deficient mice show increased emotionality and decreased rearing activity in the open-field along with neurochemical changes. Eur J Neurosci. 3:629-38.

- Dermietzel R, Hertberg EL, Kessler JA, Spray DC. (1991) Gap junctions between cultured astrocytes: immunocytochemical, molecular, and electrophysiological analysis. J Neurosci. 11:1421-32.

- Ezan P, André P, Cisternino S, Saubaméa B, Boulay AC, Doutremer S, Thomas MA, Quenech'du N, Giaume C, Cohen-Salmon M. (2012) Deletion of astroglial connexins weakens the blood-brain barrier. J Cereb Blood Flow Metab. 2012 Aug;32(8):1457-1467.

- Gendron P, Avaltroni F, Wilkinson KJ. (2008) Diffusion coefficients of several rhodamine derivatives as determined by pulsed field gradient-nuclear magnetic resonance and fluorescence correlation spectroscopy. J Fluoresc. 18(6):1093-1101.

- Genoud C, Houades V, Kraftsik R, Welker E, Giaume C. (2015) Proximity of excitatory synapses and astroglial gap junctions in layer IV of the mouse barrel cortex. Neuroscience. 291:241-249. 
- Giaume C, Fromaget C, el Aoumari A, Cordier J, Glowinski J, Gros D. (1991) Gap junctions in cultured astrocytes: single-channel currents and characterization of channelforming protein. Neuron. 6:133-43.

- Giaume C, Koulakoff A, Roux L, Holcman D, Rouach N. (2010) Astroglial networks: a step further in neuroglial and gliovascular interactions. Nat Rev Neurosci. 11:87-99.

- Hansen N (2006) The CMA evolution strategy: A comparing review. In Towards a new evolutionary computation, Lozano et al., editors. Springer-Verlag, Berlin, Heidelberg, 75102.

- Griemsmann S, Höft SP, Bedner P, Zhang J, von Staden E, Beinhauer A, Degen J, Dublin P, Cope DW, Richter N, Crunelli V, Jabs R, Willecke K, Theis M, Seifert G, Kettenmann H, Steinhäuser C. (2015) Characterization of Panglial Gap Junction Networks in the Thalamus, Neocortex, and Hippocampus Reveals a Unique Population of Glial Cells. Cereb Cortex. 25:3420-33.

- Houades V, Rouach N, Ezan P, Kirchhoff F, Koulakoff A, Giaume C. (2006) Shapes of astrocyte networks in the juvenile brain. Neuron Glia Biol. 2:3-14.

- Houades V, Koulakoff A, Ezan P, Seif I, Giaume C. (2008) Gap junction-mediated astrocytic networks in the mouse barrel cortex. J Neurosci. 28:5207-17.

- Laird DW, Lampe PD, Johnson RG. (2015) Cellular small talk. Sci Am. 312:70-77.

- Liu X, Petit JM, Ezan P, Gyger J, Magistretti P, Giaume C. (2013) The psychostimulant modafinil enhances gap junctional communication in cortical astrocytes. Neuropharmacology. 75:533-8.

- Meme W, Vandecasteele M, Giaume C, Venance L. (2009) Electrical coupling between hippocampal astrocytes in rat brain slices. Neurosci Res. 63:236-243.

- Menezes JR, Fróes MM, Moura Neto V, Lent R. (2000) Gap junction-mediated coupling in the postnatal anterior subventricular zone. Dev Neurosci. 22:34-43.

- Nagy JI, Rash JE. (2000) Connexins and gap junctions of astrocytes and oligodendrocytes in the CNS. Brain Res Brain Res Rev. 32:29-44.

- Nimmerjahn A, Kirchhoff F, Kerr JN, Helmchen F. (2004) Sulforhodamine 101 as a specific marker of astroglia in the neocortex in vivo. Nat Methods. 1:31-7.

- Pannasch U, Vargová L, Reingruber J, Ezan P, Holcman D, Giaume C, Syková E, Rouach N. (2011) Astroglial networks scale synaptic activity and plasticity. Proc Natl Acad Sci U S A. 108:8467-8472.

- Ransom BR, Giaume C. 2013. Gap junctions, hemichannels. In Neuroglia, Third Edition, H. Kettenmann, and B.R. Ransom, editors. Oxford University Press, Oxford. 292-305.

- Rouach N, Koulakoff A, Abudara V, Willecke K, Giaume C (2008) Astroglial metabolic networks sustain hippocampal synaptic transmission. Science. 322:1551-5. 
- Roux L, Benchenane K, Rothstein JD, Bonvento G, Giaume C. (2011) Plasticity of astroglial networks in olfactory glomeruli. Proc Natl Acad Sci U S A. 108:18442-18446.

- Rusakov DA. (2015) Disentangling calcium-driven astrocyte physiology. Nature Rev Neurosci. 16:226-233.

- Schnell C, Hagos Y, Hülsmann S. (2012) Active sulforhodamine 101 uptake into hippocampal astrocytes. PLoS One. 7:e49398.

- Schnell C, Shahmoradi A, Wichert SP, Mayerl S, Hagos Y, Heuer H, Rossner MJ, Hülsmann S. (2015) The multispecific thyroid hormone transporter OATP1C1 mediates cell-specific sulforhodamine 101-labeling of hippocampal astrocytes. Brain Struct Funct. 2015 Jan;220(1):193-203.

- Strohschein S, Hüttmann K, Gabriel S, Binder DK, Heinemann U, Steinhäuser C. (2011) Impact of aquaporin-4 channels on $\mathrm{K}+$ buffering and gap junction coupling in the hippocampus. Glia. 59:973-80.

- Theis M, Jauch R, Zhuo L, Speidel D, Wallraff A, Döring B, Frisch C, Söhl G, Teubner B, Euwens C, Huston J, Steinhäuser C, Messing A, Heinemann U, Willecke K. (2003) Accelerated hippocampal spreading depression and enhanced locomotory activity in mice with astrocyte-directed inactivation of connexin43. J Neurosci. 23:766-76.

- Wallraff A, Odermatt B, Willecke K, Steinhäuser C. Distinct types of astroglial cells in the hippocampus differ in gap junction coupling. Glia. 48:36-43.

- Yang S, Li WH. (2009) Assaying dynamic cell-cell junctional communication using noninvasive and quantitative fluorescence imaging techniques: LAMP and infrared-LAMP. Nat Protoc. 4:94-101.

- Yang Y, Vidensky S, Jin L, Jie C, Lorenzini I, Frankl M, Rothstein JD. Molecular comparison of GLT1+ and ALDH1L1+ astrocytes in vivo in astroglial reporter mice. Glia. 59:200-7.

- Zhong S, Du Y, Kiyoshi CM, Ma B, Alford CC, Wang Q, Yang Y, Liu X, Zhou M. (2016) Electrophysiological behavior of neonatal astrocytes in hippocampal stratum radiatum. Mol Brain 9:34. 


\begin{tabular}{|c|c|c|c|c|c|c|}
\hline \multicolumn{2}{|c|}{ Condition } & $10^{3} \mathrm{~K}_{1}\left(\mathrm{~s}^{-1}\right)$ & Amplitude $_{1}(\%)$ & $10^{3} K_{2}\left(s^{-1}\right)$ & Amplitude $_{2}(\%)$ & $n$ \\
\hline \multicolumn{2}{|c|}{ CTRL } & $36.2 \pm 11.9$ & $11.5 \pm 4.1$ & $4.90 \pm 0.66$ & $54.2 \pm 4.8$ & 5 \\
\hline \multicolumn{2}{|c|}{ CBX } & $14.7 \pm 4.0$ & $24.3 \pm 3.8$ & N/A & N/A & 4 \\
\hline \multirow{2}{*}{\multicolumn{2}{|c|}{ СХ30 ко }} & $38.0 \pm 3.4$ & $11.6 \pm 1.5$ & $4.85 \pm 0.56$ & $39.0 \pm 2.3$ & 10 \\
\hline & & 0.854 & 0.975 & 0.959 & $0.006^{* *}$ & 10 \\
\hline \multirow{2}{*}{\multicolumn{2}{|c|}{ CX43 KO }} & $45.5 \pm 5.5$ & $13.2 \pm 3.2$ & $5.53 \pm 0.81$ & $31.9 \pm 3.4$ & 12 \\
\hline & & 0.424 & 0.757 & 0.644 & $0.0024^{* *}$ & 12 \\
\hline \multirow{3}{*}{ Dble KO } & 1 comp & $\begin{array}{c}14.6 \pm 2.4 \\
0.987\end{array}$ & $\begin{array}{c}29.7 \pm 3.0 \\
0.293 \\
\end{array}$ & N/A & N/A & 6 \\
\hline & \multirow{2}{*}{2 comp } & $48.4 \pm 7.6$ & $6.8 \pm 2.0$ & $8.4 \pm 1.0$ & $26.1 \pm 2.4$ & 6 \\
\hline & & 0.389 & 0.303 & $0.0214^{*}$ & $0.0004^{* * *}$ & \\
\hline
\end{tabular}


eGFP-Aldh1L1
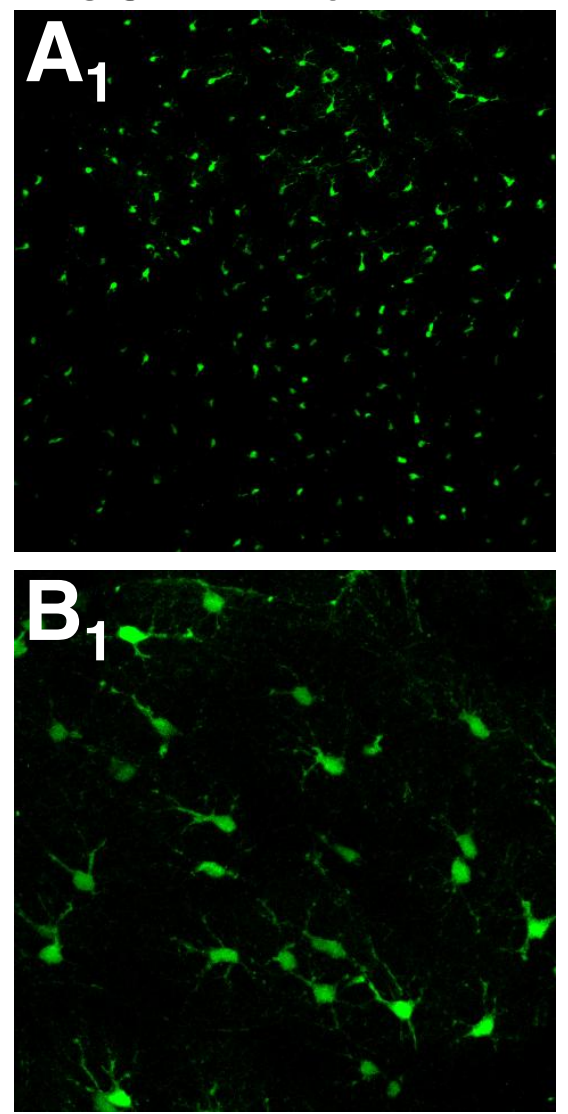

$\mathrm{C}_{1}$
SR101
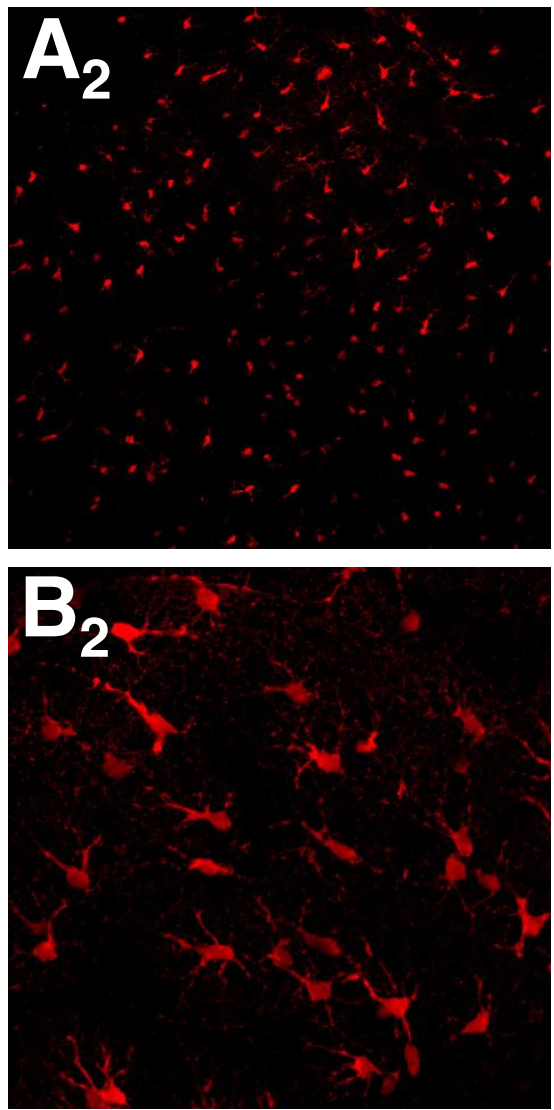

$\mathrm{C}_{2}$
Merge
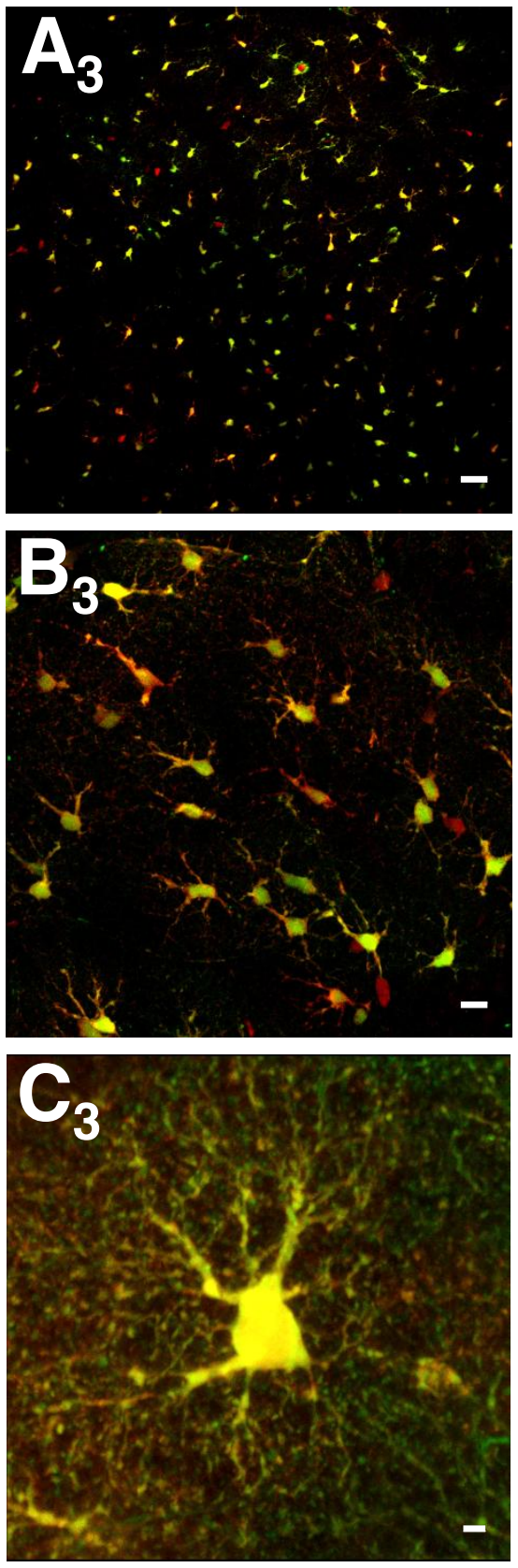
$A_{1}$ Two photons

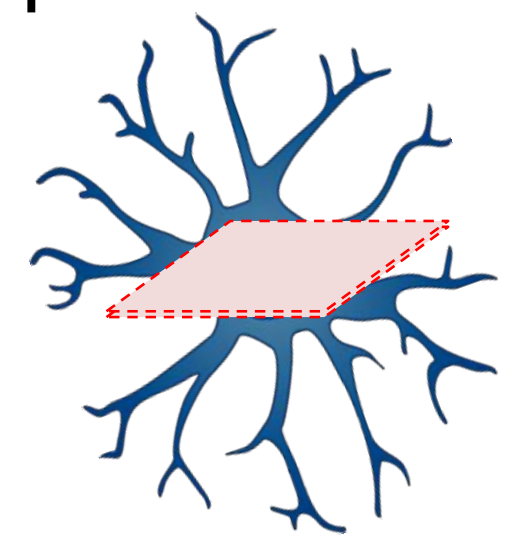

$A_{2}$ One photon
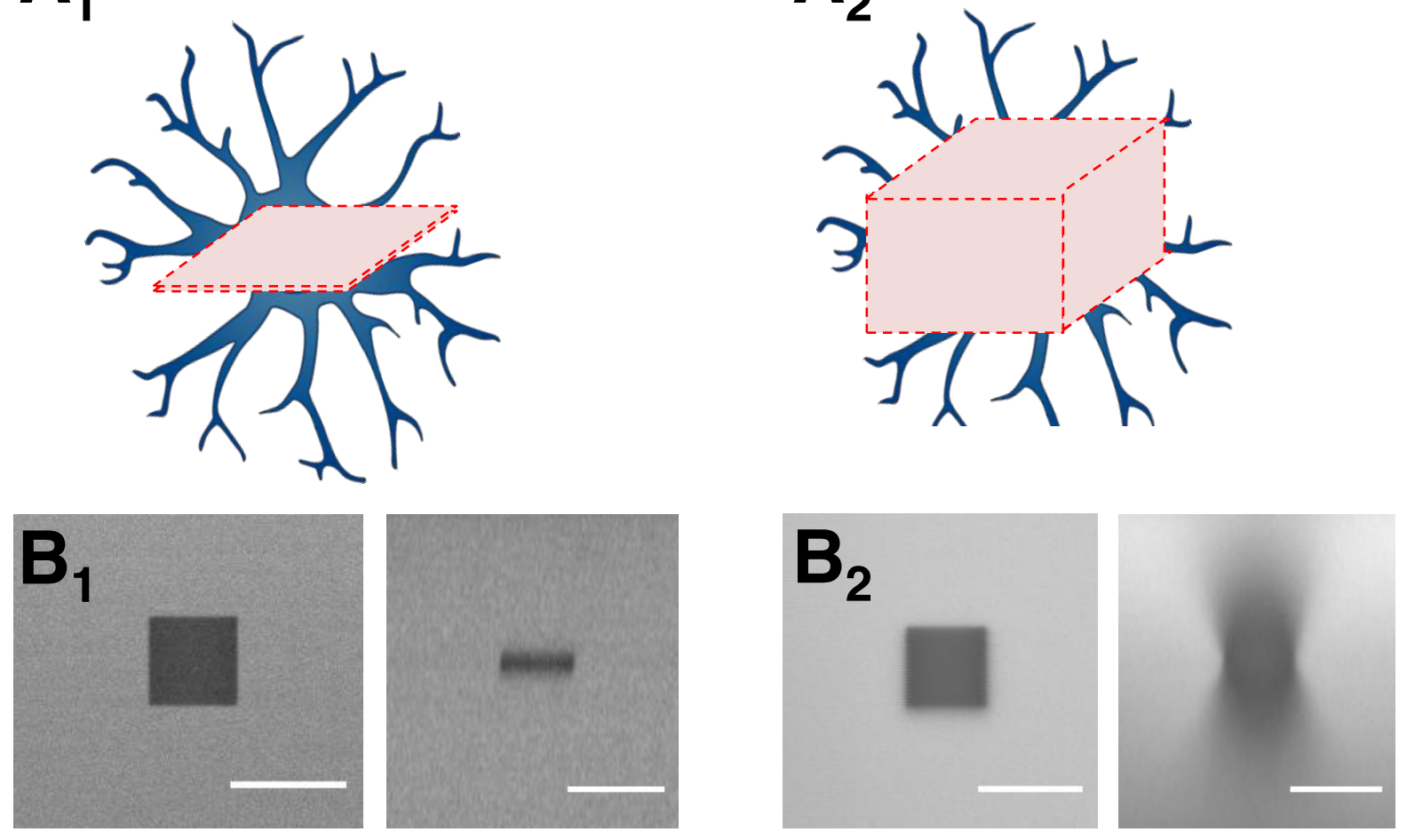

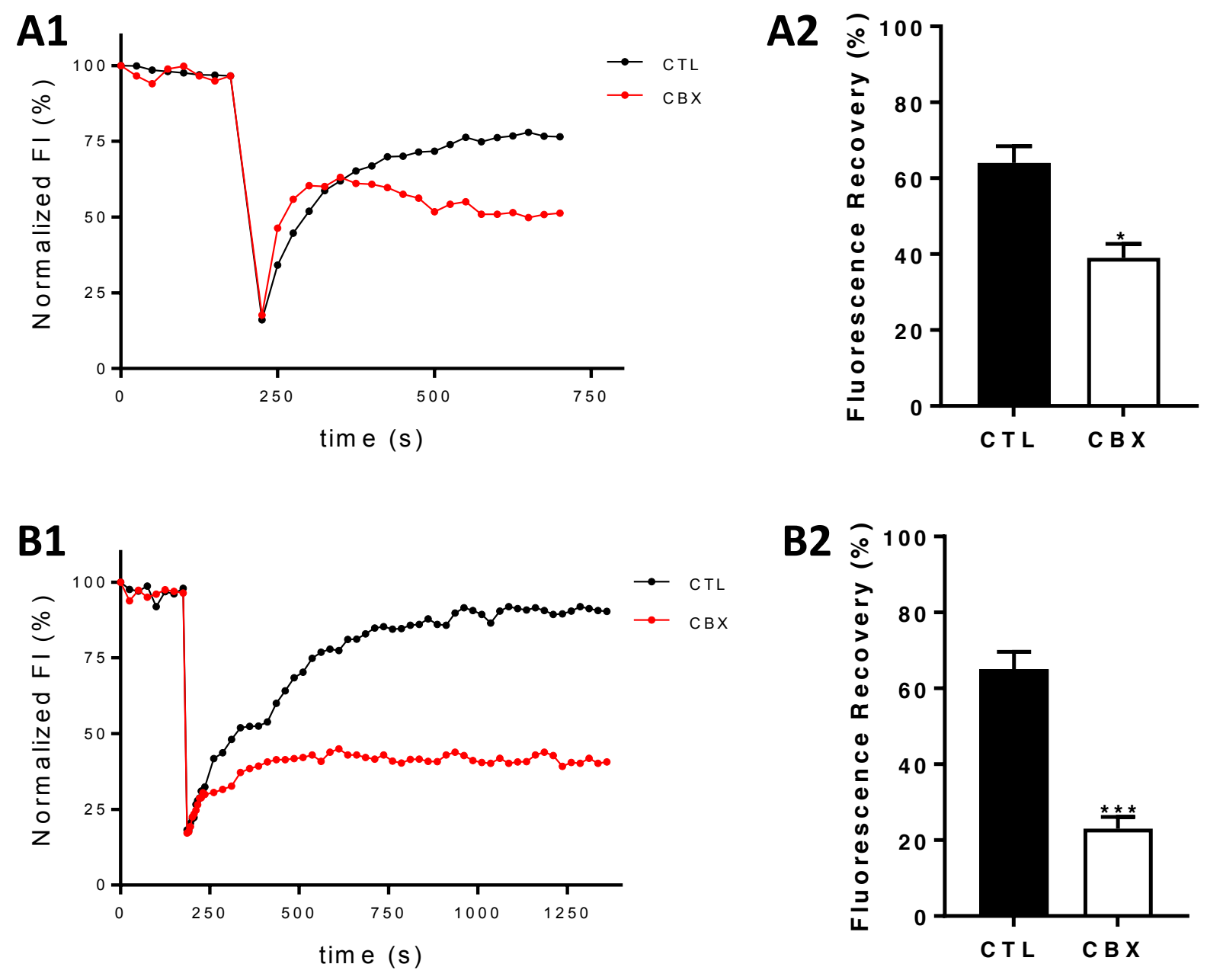

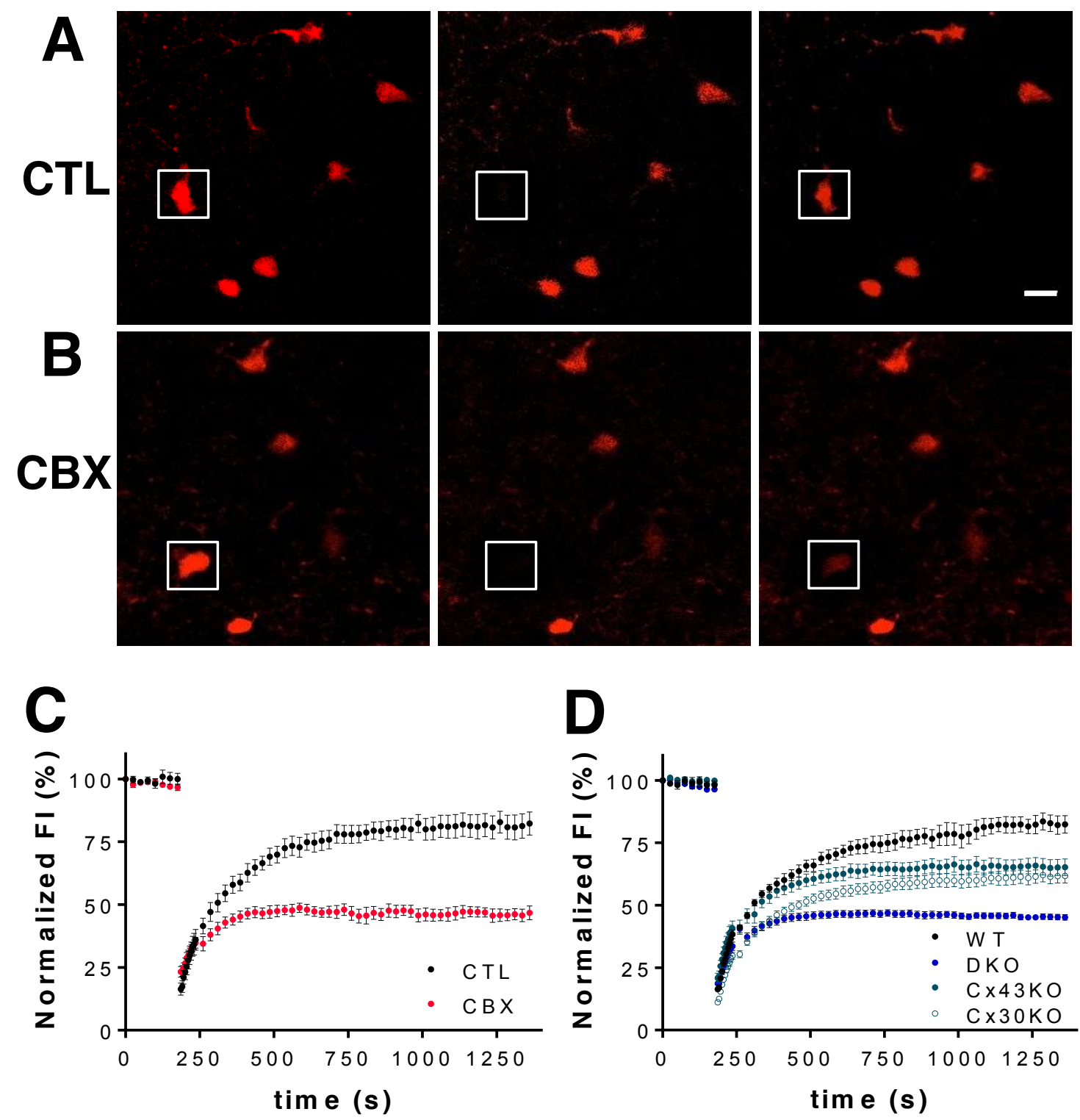

time (s)
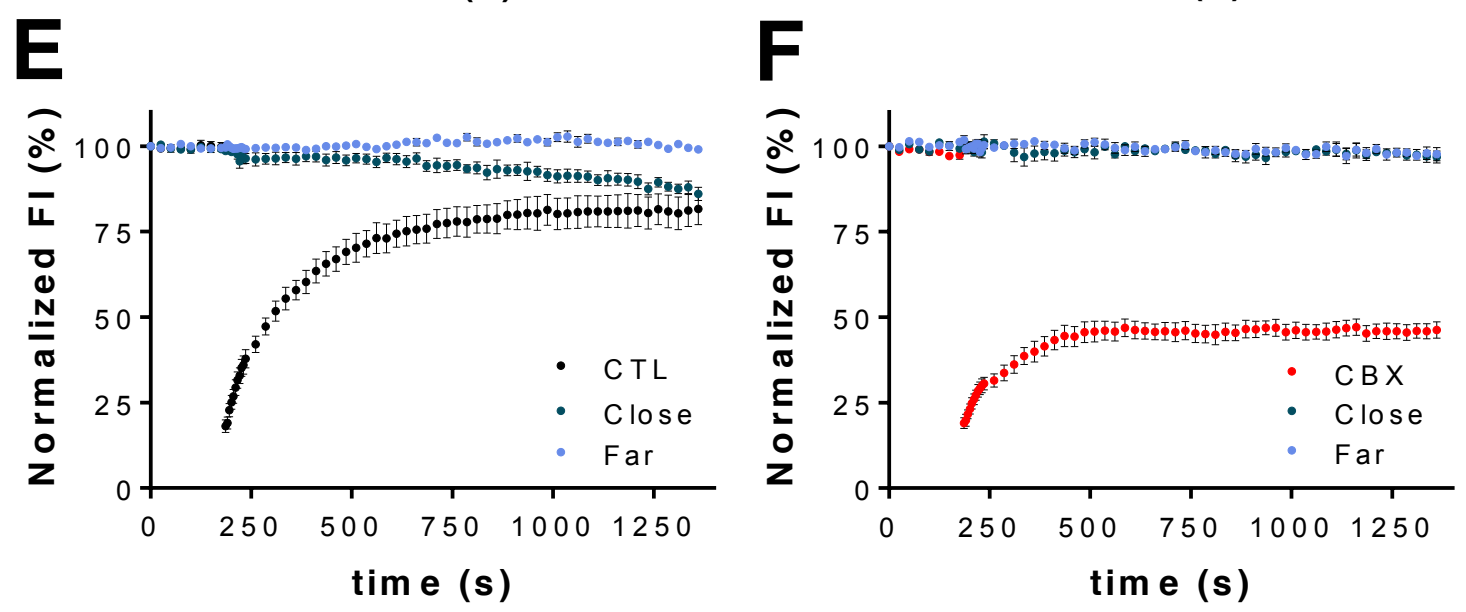
A Cell 1
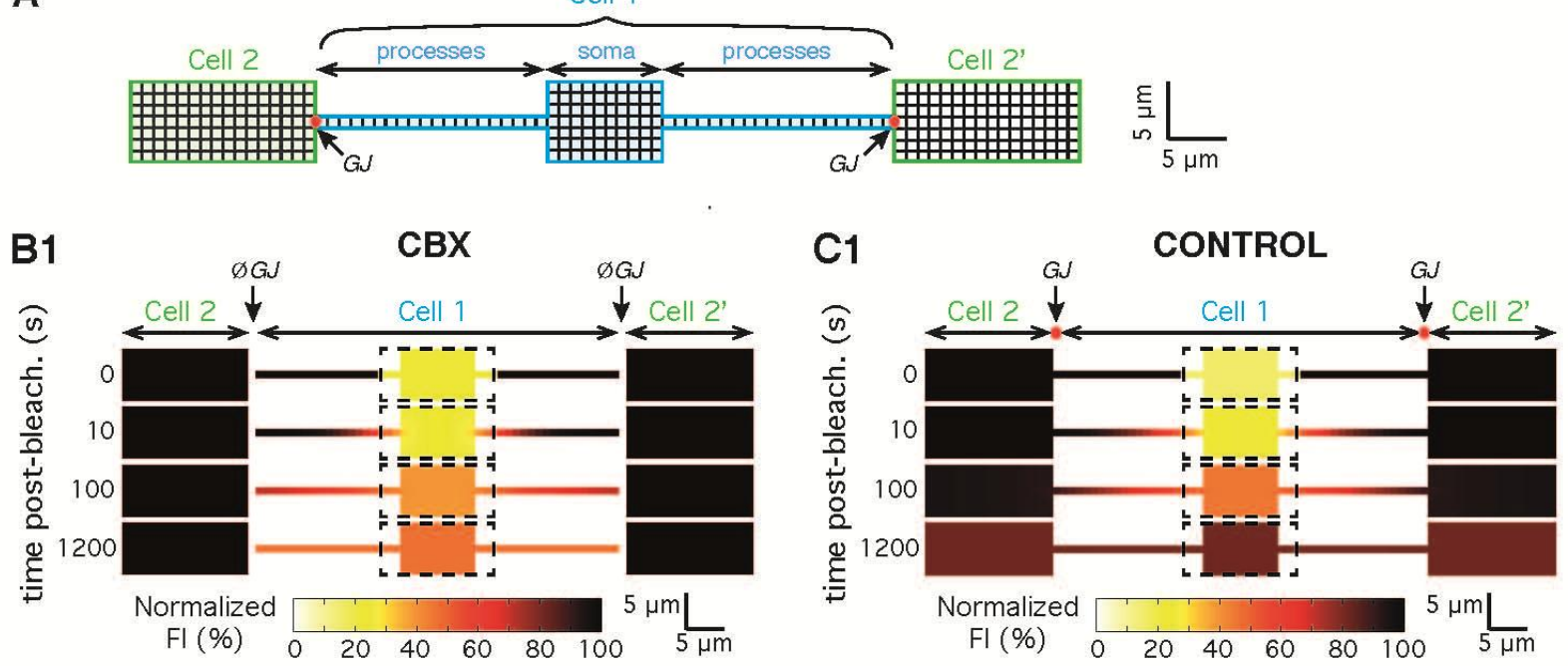

B2

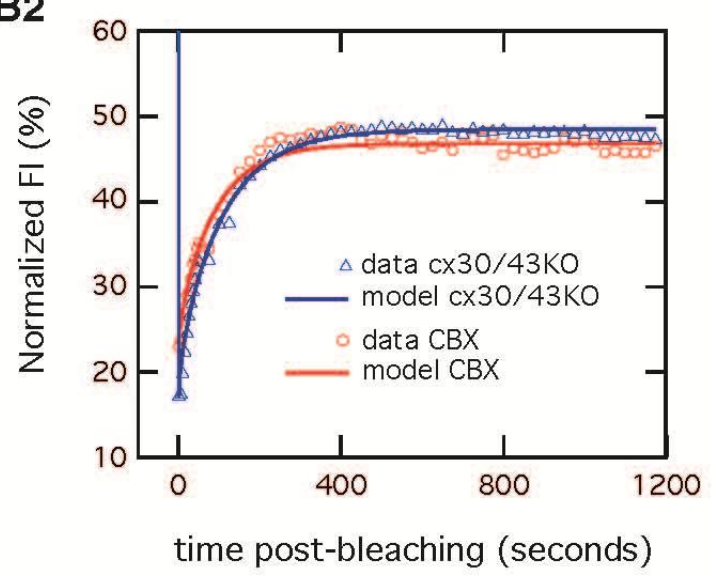

C2

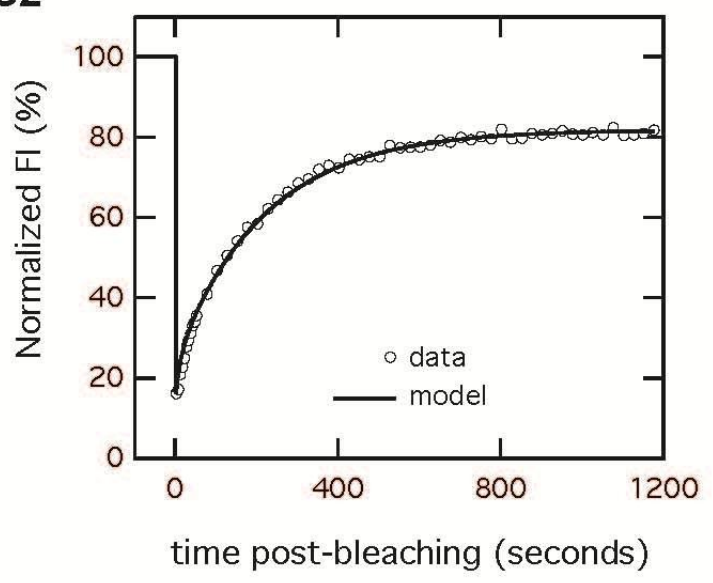




\section{B. CBX}
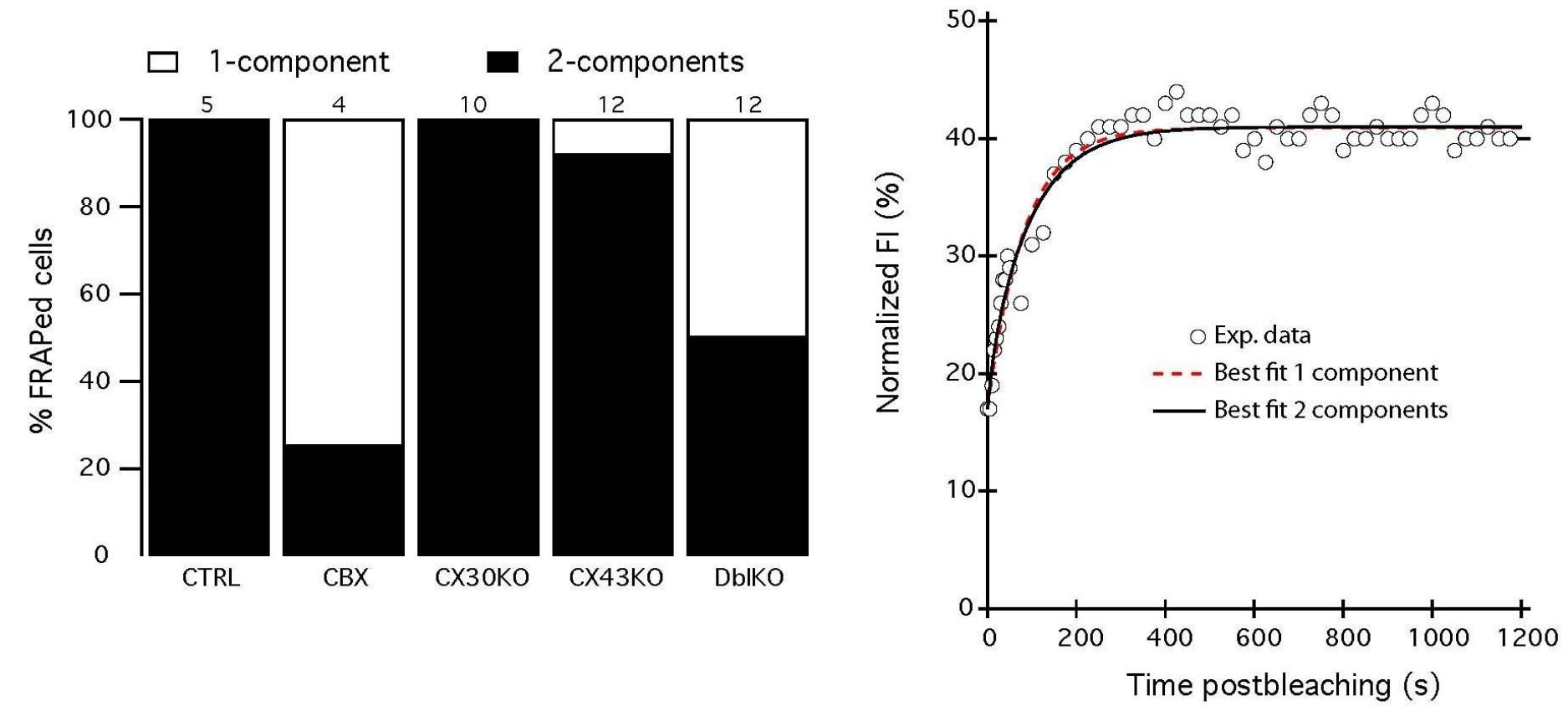

\section{CTRL}

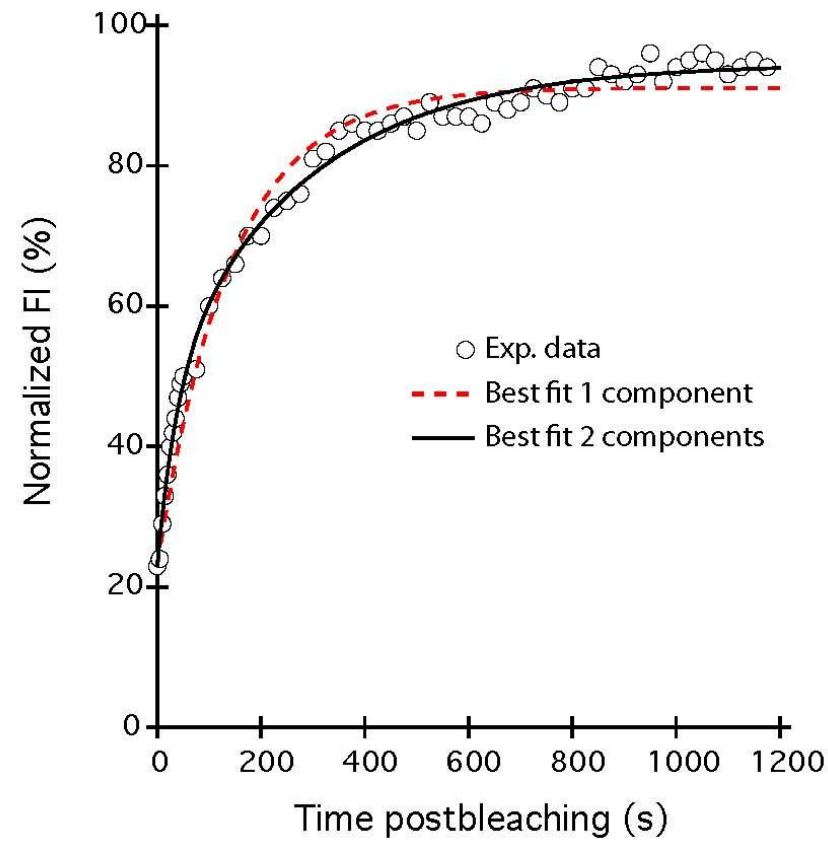

D. CX43KO

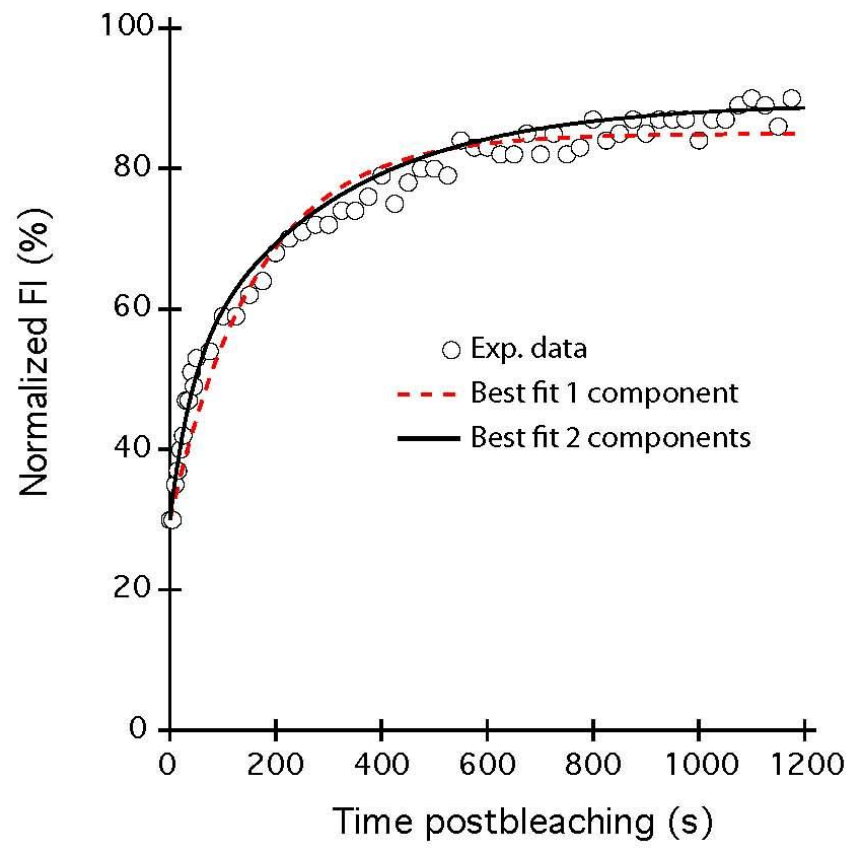

